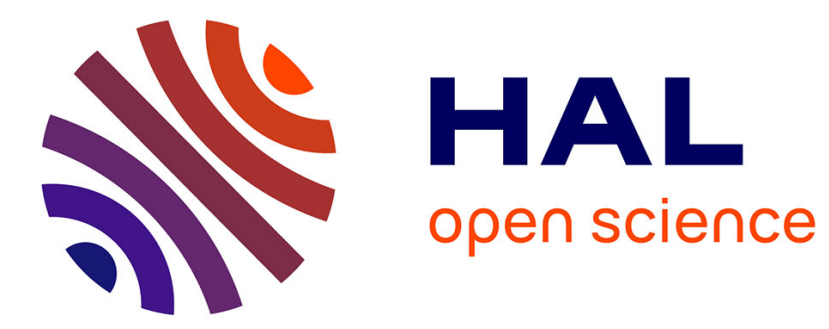

\title{
Homogeneity in the bi-limit as a tool for observer and feedback design
}

Vincent Andrieu, Laurent Praly, Alessandro Astolfi

\section{To cite this version:}

Vincent Andrieu, Laurent Praly, Alessandro Astolfi. Homogeneity in the bi-limit as a tool for observer and feedback design. 48th IEEE Conference on Decision and Control, Dec 2009, China. pp.1050 1055, 10.1109/CDC.2009.5400263 . hal-00529835

\section{HAL Id: hal-00529835 \\ https://hal.science/hal-00529835}

Submitted on 26 Oct 2010

HAL is a multi-disciplinary open access archive for the deposit and dissemination of scientific research documents, whether they are published or not. The documents may come from teaching and research institutions in France or abroad, or from public or private research centers.
L'archive ouverte pluridisciplinaire HAL, est destinée au dépôt et à la diffusion de documents scientifiques de niveau recherche, publiés ou non, émanant des établissements d'enseignement et de recherche français ou étrangers, des laboratoires publics ou privés. 


\section{Homogeneity in the bi-limit as a tool for observer and feedback design}

\author{
Vincent Andrieu
}

\author{
Laurent Praly
}

\author{
Alessandro Astolfi
}

\begin{abstract}
We introduce an extension of the notion of homogeneous approximation to make it valid both at the origin and at infinity (homogeneity in the bi-limit). Exploiting this extension, we give several results concerning stability, robustness and uniform (in the initial condition) finite time convergence for a homogeneous in the bi-limit vector field. We then introduce a homogeneous in the bi-limit observer and state-feedback for a chain of integrators. Combining these two tools we establish a global asymptotic stabilization result by output feedback for feedback and feedforward systems. We obtain also a finite time observer for globally Lipschitz system.
\end{abstract}

\section{INTRODUCTION}

The problems of designing globally convergent observers and globally asymptotically stabilizing output feedback control laws for nonlinear systems have been addressed by many authors following different routes. Many of these approaches exploit domination ideas and robustness of stability and/or convergence. In view of developing further these techniques we have introduced two new tools in [1]. The first one is an extension of the technique of homogeneous approximation to make it valid both at the origin and at infinity. The second tool is a new recursive observer design procedure for a chain of integrator. Combining these two tools we propose a global asymptotic stabilization result by output feedback for feedback and feedforward systems and a finite time convergent observer design.

To place our contribution in perspective, consider the system for which we want to design a globally asymptotically stabilizing output feedback :

$$
\dot{x}_{1}=x_{2} \quad, \quad \dot{x}_{2}=u+\delta_{2}\left(x_{2}\right) \quad, \quad y=x_{1},
$$

where :

$$
\begin{aligned}
\delta_{2}\left(x_{2}\right)=c_{0} \operatorname{sign}\left(x_{2}\right)\left|\sin \left(x_{2}\right)\right|^{q} & \\
& +c_{\infty} \operatorname{sign}\left(x_{2}\right)\left|x_{2}\right|^{p},
\end{aligned}
$$

with $p>q>0,\left(c_{0}, c_{\infty}\right)$ in $\mathbb{R}^{2}$.

In the domination approach, the nonlinear function $\delta_{2}$ is not treated per se in the design but considered as a perturbation. In this framework the output feedback controller is designed on the linear system :

$$
\dot{x}_{1}=x_{2}, \quad \dot{x}_{2}=u, \quad y=x_{1},
$$

V. Andrieu (vincent.andrieu@gmail.com) is with LAAS-CNRS, University of Toulouse, France and with université de Lyon 1, CNRS UMR 5007 LAGEP, France.

L. Praly (Laurent.Praly@ensmp.fr) is with the Centre d'Automatique et Systèmes, École des Mines de Paris, 35 Rue Saint Honoré, 77305 Fontainebleau, France.

A. Astolfi (a.astolfi@ic.ac.uk) is with the Electrical and Electronic Engineering Dept, Imperial College London, London, SW7 2AZ, UK and with Dipartimento di Informatica Sistemi e Produzione, University of Rome Tor Vergata, Via del Politecnico 1, 00133 Roma, Italy. and will be suitable for the nonlinear system (1) provided the global asymptotic stability property of the origin of the closed-loop system with $\delta_{2}\left(x_{2}\right)=0$ is robust to the nonlinear disturbance $\delta_{2}$. For instance, the design given in [13], [22] provides a linear output feedback controller which is suitable for the nonlinear system (1) when $q=1$ and $c_{\infty}=0$. This result has been extended in [21] employing a homogeneous output feedback controller which allows to deal with $p \geq 1$ and $c_{0}=0$.

Homogeneity in the bi-limit and the recursive observer design proposed in [1] allow us to deal with the case in which $c_{0} \neq 0$ and $c_{\infty} \neq 0$. In this case, the function $\delta_{2}$ is such that :

1) when $\left|x_{2}\right|$ is small and $q=1, \delta_{2}\left(x_{2}\right)$ can be approximated by $c_{0} x_{2}$ and the nonlinearity can be approximated by a linear function;

2) when $\left|x_{2}\right|$ is large, $\delta_{2}\left(x_{2}\right)$ can be approximated by $c_{\infty} x_{2}^{p}$, hence we have a polynomial growth which can be handled by a weighted homogeneous controller as in [21].

Both linear and polynomial growth are simply handled by the fact that $\delta_{3}$ becomes homogeneous as the state tends to the origin or to infinity but with different weights and degrees.

The paper is organized as follows. Section II summarizes the general properties related to homogeneity in the bi-limit established in [1]. After giving the definitions of homogeneous approximation and homogeneous in the bilimit functions and vector fields (Section II-A) we list some of their properties (Section II-B). Various results concerning stability and robustness for homogeneous in the bi-limit vector fields are given in Section II-C. In Section III we introduce a homogeneous in the bi-limit observer and state feedback for a chain of integrator. Finally, in Section IV, using the previous tools we establish results on stabilization by output feedback and finite time convergent observer.

All proofs and some examples can be found in [1] and in [3].

\section{NOTATION}

- The function $\mathfrak{H}: \mathbb{R}_{+}^{2} \rightarrow \mathbb{R}_{+}$is defined as

$$
\mathfrak{H}(a, b)=\frac{a}{1+a}[1+b] .
$$

- Given $r=\left(r_{1}, \ldots, r_{n}\right)^{T}$ in $\mathbb{R}_{+}^{n}$ and $\lambda$ in $\mathbb{R}_{+}, \lambda^{r} \diamond x=$ $\left(\lambda^{r_{1}} x_{1}, \ldots, \lambda^{r_{n}} x_{n}\right)^{T}$ is the dilation of a vector $x$ in $\mathbb{R}^{n}$ with weight $r$.

- Given $r=\left(r_{1}, \ldots, r_{n}\right)^{T}$ in $\left(\mathbb{R}_{+} \backslash\{0\}\right)^{n},|x|_{r}=$ $\left|x_{1}\right|^{\frac{1}{r_{1}}}+\ldots+\left|x_{n}\right|^{\frac{1}{r_{n}}}$ is the homogeneous norm with weight $r$ and degree 1 . 


\section{HomOgENEOUS APPROXIMATION}

\section{A. Definitions}

The use of homogeneous approximations has a long history in the study of stability of an equilibrium. It can be traced back to Lyapunov first order approximation theorem and has been pursued by many authors, for example Massera [15], Hermes [10], Rosier [24]. Similarly this technique has been used to investigate the behavior of the solutions of dynamical systems at infinity, see for instance Lefschetz in [14, IX.5] and Orsi, Praly and Mareels in [17]. In this section, we recall the definitions of homogeneous approximation at the origin and at infinity and restate some related results.

Definition 1 (Homogeneity in the 0-limit):

- A function $\phi: \mathbb{R}^{n} \rightarrow \mathbb{R}$ is said homogeneous in the 0 -limit with associated triple $\left(r_{0}, d_{0}, \phi_{0}\right)$, where $r_{0}$ in $\left(\mathbb{R}_{+} \backslash\{0\}\right)^{n}$ is the weight, $d_{0}$ in $\mathbb{R}_{+}$the degree and $\phi_{0}$ : $\mathbb{R}^{n} \rightarrow \mathbb{R}$ the approximating function, if $\phi$ is continuous, $\phi_{0}$ is continuous and not identically zero and, for each compact set $C$ in $\mathbb{R}^{n} \backslash\{0\}$ and each $\varepsilon>0$, there exists $\lambda_{0}$ such that :

$$
\max _{x \in C}\left|\frac{\phi\left(\lambda^{r_{0}} \diamond x\right)}{\lambda^{d_{0}}}-\phi_{0}(x)\right| \leq \varepsilon, \forall \quad \lambda \in\left(0, \lambda_{0}\right] .
$$

- A vector field $f=\sum_{i=1}^{n} f_{i} \frac{\partial}{\partial x_{i}}$ is said homogeneous in the 0 -limit with associated triple $\left(r_{0}, \mathfrak{d}_{0}, f_{0}\right)$, where $r_{0}$ in $\left(\mathbb{R}_{+} \backslash\{0\}\right)^{n}$ is the weight, $\mathfrak{d}_{0}$ in $\mathbb{R}$ is the degree and $f_{0}=\sum_{i=1}^{n} f_{0, i} \frac{\partial}{\partial x_{i}}$ the approximating vector field, if, for each $i$ in $\{1, \ldots, n\}, \mathfrak{d}_{0}+r_{0, i} \geq 0$ and the function $f_{i}$ is homogeneous in the 0 -limit with associated triple $\left(r_{0}, \mathfrak{d}_{0}+r_{0, i}, f_{0, i}\right)$.

This notion of local approximation of a function or of a vector field can be found in [10], [24], [5], [11].

Example 1: The function $\delta_{2}: \mathbb{R} \rightarrow \mathbb{R}$ introduced in the illustrative system (1) is homogeneous in the 0-limit with associated triple $\left(r_{0}, d_{0}, \delta_{2,0}\right)=\left(1, q, c_{0} x_{2}^{q}\right)$. Furthermore, if $q<2$ the vector field $f\left(x_{1}, x_{2}\right)=\left(x_{2}, \delta_{2}\left(x_{2}\right)\right)$ is homogeneous in the 0 -limit with associated triple :

$$
\left(r_{0}, \mathfrak{d}_{0}, f_{0}\right)=\left((2-q, 1), q-1,\left(x_{2}, c_{0} x_{2}^{q}\right)\right) .
$$

Definition 2 (Homogeneity in the $\infty$-limit):

- A function $\phi: \mathbb{R}^{n} \rightarrow \mathbb{R}$ is said homogeneous in the $\infty$-limit with associated triple $\left(r_{\infty}, d_{\infty}, \phi_{\infty}\right)$, where $r_{\infty}$ in $\left(\mathbb{R}_{+} \backslash\{0\}\right)^{n}$ is the weight, $d_{\infty}$ in $\mathbb{R}_{+}$the degree and $\phi_{\infty}: \mathbb{R}^{n} \rightarrow \mathbb{R}$ the approximating function, if $\phi$ is continuous, $\phi_{\infty}$ is continuous and not identically zero and, for each compact set $C$ in $\mathbb{R}^{n} \backslash\{0\}$ and each $\varepsilon>0$, there exists $\lambda_{\infty}$ such that :

$$
\max _{x \in C}\left|\frac{\phi\left(\lambda^{\left.r_{\infty} \diamond x\right)}\right.}{\lambda^{d_{\infty}}}-\phi_{\infty}(x)\right| \leq \varepsilon, \forall \quad \lambda \geq \lambda_{\infty} .
$$

- A vector field $f=\sum_{i=1}^{n} f_{i} \frac{\partial}{\partial x_{i}}$ is said homogeneous in the $\infty$-limit with associated triple $\left(r_{\infty}, \mathfrak{d}_{\infty}, f_{\infty}\right)$, where $r_{\infty}$ in $\left(\mathbb{R}_{+} \backslash\{0\}\right)^{n}$ is the weight, $\mathfrak{d}_{\infty}$ in $\mathbb{R}$ the degree and $f_{\infty}=\sum_{i=1}^{n} f_{\infty, i} \frac{\partial}{\partial x_{i}}$ the approximating vector field, if, for each $i$ in $\{1, \ldots, n\}, \mathfrak{d}_{\infty}+r_{\infty, i} \geq 0$ and the function $f_{i}$ is homogeneous in the $\infty$-limit with associated triple $\left(r_{\infty}, \mathfrak{d}_{\infty}+r_{\infty, i}, f_{\infty, i}\right)$.

Example $2:$ The function $\delta_{2}: \mathbb{R} \rightarrow \mathbb{R}$ given in the illustrative system (1) is homogeneous in the $\infty$-limit with associated triple $\left(r_{\infty}, d_{\infty}, \delta_{2, \infty}\right)=\left(1, p, c_{\infty} x_{2}^{p}\right)$. Furthermore, when $p<2$, the vector field $f\left(x_{1}, x_{2}\right)=\left(x_{2}, \delta_{2}\left(x_{2}\right)\right)$ is homogeneous in the $\infty$-limit with associated triple :

$$
\left(r_{\infty}, \mathfrak{d}_{\infty}, f_{\infty}\right)=\left((2-p, 1), p-1,\left(x_{2}, c_{\infty} x_{2}^{p}\right)\right) .
$$

Definition 3 (Homogeneity in the bi-limit): A function $\phi: \mathbb{R}^{n} \rightarrow \mathbb{R}$ (or a vector field $f: \mathbb{R}^{n} \rightarrow \mathbb{R}^{n}$ ) is said homogeneous in the bi-limit if it is homogeneous in the 0 limit and homogeneous in the $\infty$-limit.

Remark 1 : If a function $\phi$ (respectively a vector field $f$ ) is homogeneous in the bi-limit, then the approximating function $\phi_{0}$ or $\phi_{\infty}$ (resp. the approximating vector field $f_{0}$ or $f_{\infty}$ ) is homogeneous in the standard sense (with the same weight and degree).

Example 3 : As a consequence of Examples 1 and 2, the vector field $f\left(x_{1}, x_{2}\right)=\left(x_{2}, \delta_{2}\left(x_{2}\right)\right)$ is homogeneous in the bi-limit with associated triples given in (5) and (6) as long as $0<q<p<2$.

\section{B. Properties of homogeneous approximations}

Since all the properties presented in this paragraph concern in the same way homogeneity in 0-limit and in the $\infty$-limit, we introduce the letter " $b$ ", which stands for "0" or " $\infty "$.

To begin with note that the weight and degree of an homogeneous in the $b$-limit function are not uniquely defined. Indeed, if $\phi$ is homogeneous in the $b$-limit with associated triple $\left(r_{b}, d_{b}, \phi_{b}\right)$, then it is also homogeneous in the $b$-limit with associated triple $\left(k r_{b}, k d_{b}, \phi_{b}\right)$ for all $k>0$. (Simply change $\lambda$ in $\lambda^{k}$.)

Here is a list of properties established in [1].

Proposition 1 (Basic properties): Let $\phi$ and $\zeta$ be two functions homogeneous in the $b$-limit, with weights $r_{\phi, b}$ and $r_{\zeta, b}$, degrees $d_{\phi, b}$ and $d_{\zeta, b}$, and approximating functions $\phi_{b}$ and $\zeta_{b}$. The following hold.

P1 : If there exists $k$ in $\mathbb{R}_{+}$such that $k r_{\phi, b}=r_{\zeta, b}$ then the function $x \mapsto \phi(x) \zeta(x)$ is homogeneous in the $b$-limit with weight $r_{\zeta, b}$, degree $k d_{\phi, b}+d_{\zeta, b}$ and approximating function $x \mapsto \phi_{b}(x) \zeta_{b}(x)$.

P2 : If, for each $j$ in $\{1, \ldots, n\}, \frac{d_{\phi, b}}{r_{\phi, b, j}}<\frac{d_{\zeta, b}}{r_{\zeta, b, j}}$, then the function $x \mapsto \phi(x)+\zeta(x)$ is homogeneous in the $b$ limit with degree $d_{\phi, b}$, weight $r_{\phi, b}$ and approximating function $x \mapsto \phi_{b}(x)$. In this case we say that the function $\phi$ dominates the function $\zeta$ in the $b$-limit.

P3 : If the function $\phi_{b}+\zeta_{b}$ is not identically zero and, for each $j$ in $\{1, \ldots, n\}, \frac{d_{\phi, b}}{r_{\phi, b, j}}=\frac{d_{\zeta, b}}{r_{\zeta, b, j}}$, then the function $x \mapsto \phi(x)+\zeta(x)$ is homogeneous in the $b$-limit with degree $d_{\phi, b}$, weight $r_{\phi, b}$ and approximating function $x \mapsto \phi_{b}(x)+\zeta_{b}(x)$.

Proposition 2 (Composition function): If $\phi: \mathbb{R}^{n} \rightarrow \mathbb{R}$ and $\zeta: \mathbb{R} \rightarrow \mathbb{R}$ are homogeneous in the $b$-limit functions, 
with weights $r_{\phi, b}$ and $r_{\zeta, b}$, degrees $d_{\phi, b}>0$ and $d_{\zeta, b} \geq$ 0 , and approximating functions $\phi_{b}$ and $\zeta_{b}$, then $\zeta \circ \phi$ is homogeneous in the $b$-limit with weight $r_{\phi, b}$, degree $\frac{d_{\zeta, b} d_{\phi, b}}{r_{\zeta, b}}$, and approximating function $\zeta_{b} \circ \phi_{b}$.

Proposition 3 (Inverse function): Let $\phi: \mathbb{R} \rightarrow \mathbb{R}$ be a bijective homogeneous in the $b$-limit function with associated triple $\left(1, d_{b}, \varphi_{b} x^{d_{b}}\right)$ with $\varphi_{b} \neq 0$ and $d_{b}>0$. Then the inverse function $\phi^{-1}: \mathbb{R} \rightarrow \mathbb{R}$ is a homogeneous in the $b$-limit function with associated triple $\left(1, \frac{1}{d_{b}},\left(\frac{x}{\varphi_{b}}\right)^{\frac{1}{d_{b}}}\right)$.

Proposition 4 (Integral function): If the function $\phi$ : $\mathbb{R}^{n} \rightarrow \mathbb{R}$ is homogeneous in the $b$-limit with associated triple $\left(r_{b}, d_{b}, \phi_{b}\right)$, then the function $\Phi_{i}(x)=$ $\int_{0}^{x_{i}} \phi\left(x_{1}, \ldots, x_{i-1}, s, x_{i+1}, \ldots, x_{n}\right) d s$ is homogeneous in the $b$-limit with associated triple $\left(r_{b}, d_{b}+r_{b, i}, \Phi_{i, b}\right)$, with $\Phi_{i, b}(x)=\int_{0}^{x_{i}} \phi_{b}\left(x_{1}, \ldots, x_{i-1}, s, x_{i+1}, \ldots, x_{n}\right) d s$.

Proposition 5: Let $\phi: \mathbb{R}^{n} \rightarrow \mathbb{R}$ and $\zeta: \mathbb{R}^{n} \rightarrow \mathbb{R}_{+}$ be two homogeneous in the bi-limit functions with the same weights $r_{0}$ and $r_{\infty}$, degrees $d_{\phi, 0}, d_{\phi, \infty}$ and $d_{\zeta, 0}, d_{\zeta, \infty}$, and approximating functions $\phi_{0}, \phi_{\infty}$ and $\zeta_{0}, \zeta_{\infty}$. If the degrees satisfy $d_{\phi, 0} \geq d_{\zeta, 0}$ and $d_{\phi, \infty} \leq d_{\zeta, \infty}$ and the functions $\zeta, \zeta_{0}$ and $\zeta_{\infty}$ are positive definite then there exists a positive real number $c$ satisfying :

$$
\phi(x) \leq c \zeta(x) \quad, \forall x \in \mathbb{R}^{n} .
$$

\section{Stability and homogeneous approximation}

A very basic property of asymptotic stability is its robustness. This fact was already known to Lyapunov who proposed his second method, (local) asymptotic stability of an equilibrium is established by looking at the first order approximation of the system. The case of local homogeneous approximations of higher degree has been investigated by Massera [15], Hermes [10] and Rosier [24].

Proposition 6 ([24]): Consider a homogeneous in the 0limit vector field $f: \mathbb{R}^{n} \rightarrow \mathbb{R}^{n}$ with associated triple $\left(r_{0}, \mathfrak{d}_{0}, f_{0}\right)$. If the origin of the system :

$$
\dot{x}=f_{0}(x)
$$

is locally asymptotically stable then the origin of

$$
\dot{x}=f(x)
$$

is locally asymptotically stable.

Consequently, a natural strategy to ensure local asymptotic stability of an equilibrium of a system is to design a stabilizing homogeneous control law for the homogeneous approximation in the 0-limit (see [10], [12], [8] for instance).

In the context of homogeneity in the $\infty$-limit, we have the following result.

Proposition 7: Consider a homogeneous in the $\infty$-limit vector field $f: \mathbb{R}^{n} \rightarrow \mathbb{R}^{n}$ with associated triple $\left(r_{\infty}, \mathfrak{d}_{\infty}, f_{\infty}\right)$. If the origin of the system

$$
\dot{x}=f_{\infty}(x)
$$

is globally asymptotically stable then there exists an invariant compact subset of $\mathbb{R}^{n}$, denoted $\mathcal{C}_{\infty}$, which is globally asymptotically stable for the system :

$$
\dot{x}=f(x) \text {. }
$$

As in the case of homogeneity in the 0-limit, this property can be used to design a feedback ensuring boundedness of solutions.

Example 4: Consider the system (1) with $0<q<p<2$ and the control law :

$u=-\frac{1}{2-p} x_{1}^{\frac{p-1}{2-p}} x_{2}-x_{1}^{\frac{p}{2-p}}-c_{\infty} x_{2}^{p}-\left(x_{2}+x_{1}^{\frac{1}{2-p}}\right)^{p}$.

This control law is such that the closed loop vector field is homogeneous in the $\infty$-limit with degree $\mathfrak{d}_{\infty}=p-1$, weight $(2-p, 1)$ and associated vector field $f_{\infty}\left(x_{1}, x_{2}\right)=$ $\left(x_{2},-\frac{1}{2-p} x_{1}^{\frac{p-1}{2-p}} x_{2}-x_{1}^{\frac{p}{2-p}}-\left(x_{2}+x_{1}^{\frac{1}{2-p}}\right)^{p}\right)^{T}$. For the homogeneous Lyapunov function of degree two :

$$
V_{\infty}\left(x_{1}, x_{2}\right)=\frac{2-p}{2}\left|x_{1}\right|^{\frac{2}{2-p}}+\frac{1}{2}\left|x_{2}+x_{1}^{\frac{1}{2-p}}\right|^{2},
$$

we get :

$$
\frac{\partial V_{\infty}}{\partial x}(x) f_{\infty}(x)=-\left|x_{1}\right|^{\frac{p+1}{2-p}}-\left|x_{2}+x_{1}^{\frac{1}{2-p}}\right|^{p+1} .
$$

It follows that the control law (7) guarantees boundedness of the solutions of the closed loop system. Furthermore, boundedness of solutions is preserved in the presence of any perturbation which does not change the approximating homogeneous function in the $\infty$-limit, i.e. in the presence of perturbations which are negligible with respect to the dominant homogeneous part (see Proposition 1.P2 in Section II-B).

The key step in the proof of Propositions 6 and 7 is the converse Lyapunov theorem given by Rosier in [24]. This result can also be extended to the case of homogeneity in the bi-limit.

Theorem 1 (Homogeneous in the bi-limit Lyapunov functions): Consider a homogeneous in the bi-limit vector field $f: \mathbb{R}^{n} \rightarrow \mathbb{R}^{n}$, with associated triples $\left(r_{\infty}, \mathfrak{d}_{\infty}, f_{\infty}\right)$ and $\left(r_{0}, \mathfrak{d}_{0}, f_{0}\right)$ such that the origins of the systems :

$$
\dot{x}=f(x) \quad, \quad \dot{x}=f_{\infty}(x) \quad, \quad \dot{x}=f_{0}(x)
$$

are globally asymptotically stable equilibria. Let $d_{V_{\infty}}$ and $d_{V_{0}}$ be real numbers such that $d_{V_{\infty}}>\max _{1 \leq i \leq n} r_{\infty, i}$ and $d_{V_{0}}>\max _{1 \leq i \leq n} r_{0, i}$. Then there exists a $C^{1}$, positive definite and proper function $V: \mathbb{R}^{n} \rightarrow \mathbb{R}_{+}$ such that, for each $i$ in $\{1, \ldots, n\}$, the functions $x \mapsto$ $\frac{\partial V}{\partial x_{i}}$ is homogeneous in the bi-limit with associated triples $\left(r_{0}, d_{V_{0}}-r_{0, i}, \frac{\partial V_{0}}{\partial x_{i}}\right)$ and $\left(r_{\infty}, d_{V_{\infty}}-r_{\infty, i}, \frac{\partial V_{\infty}}{\partial x_{i}}\right)$ and the functions $x \mapsto \frac{\partial V}{\partial x}(x) f(x), x \mapsto \frac{\partial V_{0}}{\partial x}(x) f_{0}(x)$ and $x \mapsto$ $\frac{\partial V_{\infty}}{\partial x}(x) f_{\infty}(x)$ are negative definite.

A direct consequence of this result is an Input-to-State Stability (ISS) property with respect to disturbances (see [26]). To illustrate this property, consider the system with exogenous disturbance $\delta=\left(\delta_{1}, \ldots, \delta_{m}\right)$ in $\mathbb{R}^{m}$ :

$$
\dot{x}=f(x, \delta),
$$


with $f: \mathbb{R}^{n} \times \mathbb{R}^{m}$ a continuous vector field homogeneous in the bi-limit with associated triples $\left(\mathfrak{d}_{0},\left(r_{0}, \mathfrak{r}_{0}\right), f_{0}\right)$ and $\left(\mathfrak{d}_{\infty},\left(r_{\infty}, \mathfrak{r}_{\infty}\right), f_{\infty}\right)$ where $\mathfrak{r}_{0}$ and $\mathfrak{r}_{\infty}$ in $\left(\mathbb{R}_{+} \backslash\{0\}\right)^{m}$ are the weights associated to the disturbance $\delta$.

Corollary 1 (ISS Property): If the origins of the systems :

$$
\dot{x}=f(x, 0) \quad, \quad \dot{x}=f_{0}(x, 0) \quad, \quad \dot{x}=f_{\infty}(x, 0)
$$

are globally asymptotically stable equilibria, then the function $V$ given by Theorem 1 satisfies $^{1}$ for all $\delta=\left(\delta_{1}, \ldots, \delta_{m}\right)$ in $\mathbb{R}^{m}$ and $x$ in $\mathbb{R}^{n}$ :

$$
\begin{aligned}
\frac{\partial V}{\partial x}(x) f(x, \delta) \leq & -c_{V} \mathfrak{H}\left(V(x)^{\frac{d_{V_{0}}+d_{0}}{d_{V_{0}}}}, V(x)^{\frac{d_{V_{\infty}}+d_{\infty}}{d_{V_{\infty}}}}\right) \\
& +c_{\delta} \sum_{j=1}^{m} \mathfrak{H}\left(\left|\delta_{j}\right|^{\frac{d_{V_{0}}+d_{0}}{\mathrm{r}_{0, j}}},\left|\delta_{j}\right|^{\frac{d_{V_{\infty}}+d_{\infty}}{\mathrm{r}_{\infty, j}}}\right),
\end{aligned}
$$

where $c_{V}$ and $c_{\delta}$ are positive real numbers.

In other words, system (9) with $\delta$ as input is ISS.

Finally, we have also the following small-gain result for homogeneous in the bi-limit vector fields.

Corollary 2 (Small-Gain): Under the hypotheses of Corollary 1, there exists a real number $c_{G}>0$ such that, for each class $\mathcal{K}$ function $\gamma_{z}$ and $\mathcal{K} \mathcal{L}$ function $\beta_{\delta}$, there exists a class $\mathcal{K} \mathcal{L}$ function $\beta_{x}$ such that, for each function $t \in[0, T) \mapsto(x(t), \delta(t), z(t)), T \leq+\infty$, with $x C^{1}$ and $\delta$ and $z$ continuous, which satisfies, both (9) on $[0, T)$ and, for all $0 \leq s \leq t \leq T$,

$$
\begin{aligned}
|z(t)| \leq \max \{ & \left.\beta_{\delta}(|z(s)|, t-s), \sup _{s \leq \kappa \leq t} \gamma_{z}(|x(\kappa)|)\right\}(11) \\
\left|\delta_{i}(t)\right| \leq \max \{ & \left\{\beta_{\delta}(|z(s)|, t-s),\right. \\
& \left.c_{G} \sup _{s \leq \kappa \leq t}\left\{\mathfrak{H}\left(\left.|x(\kappa)|\right|_{r_{0}, i} ^{\mathfrak{r}_{0}},|x(\kappa)|_{r_{\infty}, i}^{\mathfrak{r}_{\infty}}\right)\right\}\right\},
\end{aligned}
$$

we have

$$
|x(t)| \leq \beta_{x}(|(x(s), z(s))|, t-s) \quad 0 \leq s \leq t \leq T .
$$

Example 5 : An interesting case which can be dealt with by Corollary 2 is when the $\delta_{i}$ 's are outputs of auxiliary systems with state $z_{i}$ in $\mathbb{R}^{n_{i}}$, i.e :

$$
\delta_{i}(t):=\delta_{i}\left(z_{i}(t), x(t)\right) \quad, \quad \dot{z}_{i}=g_{i}\left(z_{i}, x\right) .
$$

It can be checked that the bounds (12) and (11) are satisfied by all the solutions of (9) and (14) if there exist positive definite and radially unbounded functions and $\omega_{3}$, a positive real number $\epsilon$ in $(0,1)$ such that for all $x$ in $\mathbb{R}^{n}$, for all $i$ in $\{1, \ldots, m\}$ and $z_{i}$ in $\mathbb{R}^{n_{i}}$, we have :

$$
\begin{aligned}
& \left|\delta_{i}\left(z_{i}, x\right)\right| \leq \omega_{1}(x)+\omega_{2}\left(Z_{i}\left(z_{i}\right)\right) \\
& \frac{\partial Z_{i}}{\partial z_{i}}\left(z_{i}\right) g_{i}\left(z_{i}, x\right) \leq-Z_{i}\left(z_{i}\right)+\omega_{3}(|x|), \\
& \omega_{1}(x)+\omega_{2}\left([1+\epsilon] \omega_{3}(|x|)\right) \leq c_{G} \mathfrak{H}\left(|x|_{r_{0}}^{\mathfrak{r}_{0, i}},|x|_{r_{\infty}}^{\mathfrak{r}_{\infty}, i}\right) .
\end{aligned}
$$

Another important result exploiting Theorem 1 deals with finite time convergence of solutions to the origin when this is a globally asymptotically stable equilibrium (see [6]). It

\footnotetext{
${ }^{1}$ The function $\mathfrak{H}$ is defined in (4).
}

is well known that when the origin of the homogeneous approximation in the 0-limit is globally asymptotically stable and with a strictly negative degree then solutions converge to the origin in finite time (see [7]). We extend this result by showing that if, furthermore the origin of the homogeneous approximation in the $\infty$-limit is globally asymptotically stable with strictly positive degree then the convergence time does not depend on the initial condition. We call this property finite time convergence uniform in the initial condition or simply uniform finite time convergence.

Corollary 3 (Uniform Finite Time Convergence): Under the hypotheses of Theorem 1 , if we have $\mathfrak{d}_{\infty}>0>\mathfrak{d}_{0}$, then all solutions of the system $\dot{x}=f(x)$ converge in finite time to the origin, uniformly in the initial condition.

\section{HomogeneOUS IN THE BI-LIMIT TOOLS FOR A CHAIN OF INTEGRATORS}

The notion of homogeneity in the bi-limit is instrumental to introduce an observer and a state feedback for a chain of integrator. Throughout this section we consider an input output system given as a chain of integrators, with state $\mathfrak{X}=$ $\left(\mathcal{X}_{1}, \ldots, \mathcal{X}_{n}\right)$ in $\mathbb{R}^{n}$, input $u$ in $\mathbb{R}$ and output $y$ in $\mathbb{R}$, namely :

$$
\dot{\mathfrak{X}}=\mathcal{S} \mathfrak{X}+B u, y=C \mathfrak{X}
$$

where $\mathcal{S}$ is the shift matrix of order $n$, i.e. $\mathcal{S} \mathfrak{X}=$ $\left(\mathcal{X}_{2}, \ldots, \mathcal{X}_{n}, 0\right)^{T}, \quad B=(0, \ldots, 0,1)^{T}$, and $C=$ $(1,0, \ldots, 0)$. By selecting arbitrary vector field degrees $\mathfrak{d}_{0}$ and $\mathfrak{d}_{\infty}$ in $\left(-1, \frac{1}{n-1}\right)$, we see that, to possibly obtain homogeneity in the bi-limit of the vector field associated with (15) with some feedback for $u$, we must choose the weights $r_{0}=\left(r_{0,1}, \ldots, r_{0, n}\right)$ and $r_{\infty}=\left(r_{\infty, 1}, \ldots, r_{\infty, n}\right)$ as :

$$
r_{b, n}=1, \quad r_{b, i}=r_{b, i+1}-\mathfrak{d}_{b}=1-\mathfrak{d}_{b}(n-i),
$$

where the letter " $b$ " stands for " 0 " or " $\infty$ ".

\section{A. Homogeneous in the bi-limit Observer}

The goal of this section is to introduce a global homogeneous in the bi-limit observer for the system (15). The design presented in [1] follows a recursive method.

The observer is given by the system :

$$
\dot{\hat{\mathfrak{X}}}=\mathcal{S} \hat{\mathfrak{X}}+B u+K\left(\hat{\mathcal{X}}_{1}-y\right)
$$

with state $\hat{\mathfrak{X}}=\left(\hat{\mathcal{X}}_{1}, \ldots, \hat{\mathcal{X}}_{n}\right)$, and where $K: \mathbb{R} \rightarrow \mathbb{R}^{n}$ is a homogeneous in the bi-limit vector field with weights $r_{0}$ and $r_{\infty}$, and degrees $\mathfrak{d}_{0}$ and $\mathfrak{d}_{\infty}$. If we introduce the estimation error vector $E$ in $\mathbb{R}^{n}$ defined as $E=\hat{\mathfrak{X}}-\mathfrak{X}$, the output injection vector field $K$ has to be selected such that the origin is a globally asymptotically stable equilibrium for the system :

$$
\dot{E}=\mathcal{S} E+K\left(e_{1}\right) \quad, \quad E=\left(e_{1}, \ldots e_{n}\right)^{T},
$$

and also for its homogeneous approximations.

Theorem 2 (Homogeneous in the bi-limit observer):

With $\mathfrak{d}_{0}, \mathfrak{d}_{\infty}, r_{0}$ and $r_{\infty}$ satisfying the constraints given in the introduction of this section, there exists a homogeneous 
in the bi-limit vector field $K: \mathbb{R} \rightarrow \mathbb{R}^{n}$, with associated triples $\left(r_{0}, \mathfrak{d}_{0}, K_{0}\right)$ and $\left(r_{\infty}, \mathfrak{d}_{\infty}, K_{\infty}\right)$, such that, the origin is a globally asymptotically stable equilibrium for the system (18) and its homogeneous approximation:

$$
\dot{E}=\mathcal{S} E+K_{0}\left(e_{1}\right) \quad, \quad \dot{E}=\mathcal{S} E+K_{\infty}\left(e_{1}\right) .
$$

\section{B. Homogeneous in the bi-limit state feedback}

It is well-known that the system (15) can be rendered homogeneous by using a stabilizing homogeneous state feedback which can be designed by backstepping (see [19], [16], [21], [11] for instance). We show in this section that this property can be extended to the case of homogeneity in the bi-limit.

Theorem 3 (Homogeneous in the bi-limit state-feedback): With $\mathfrak{d}_{0}, \mathfrak{d}_{\infty}, r_{0}$ and $r_{\infty}$ satisfying the constraints given in the introduction of this section, there exits a homogeneous in the bi-limit function $\phi: \mathbb{R}^{n} \rightarrow \mathbb{R}$ with associated triples $\left(r_{0}, \mathfrak{d}_{0}+1, \phi_{0}\right)$ and $\left(r_{\infty}, \mathfrak{d}_{\infty}+1, \phi_{\infty}\right)$ such that, with $u=\phi(\mathfrak{X})$, the origin is a globally asymptotically stable equilibrium of the systems (15) and its homogeneous approximations, i.e.

$$
\dot{\mathfrak{X}}=\mathcal{S} \mathfrak{X}+B \phi_{0}(\mathfrak{X}) \quad, \quad \dot{\mathfrak{X}}=\mathcal{S} \mathfrak{X}+B \phi_{\infty}(\mathfrak{X})
$$

\section{Homogeneous in the bi-limit output feedback}

With the observer and the state feedback we can derive a result on stabilization by output feedback for the origin of system (15). The output feedback is obtained following an observer-controller approach and is given as:

$$
\dot{\hat{\mathfrak{X}}}=\mathcal{S} \hat{\mathfrak{X}}+B \phi(\hat{\mathfrak{X}})+K\left(\hat{\mathcal{X}}_{1}-y\right), \quad u=\phi(\hat{\mathfrak{X}})
$$

with $\hat{\mathfrak{X}}$ in $\mathbb{R}^{n}$ and where $\phi$ and $K$ are the homogeneous functions obtained from Theorem 3 and 2:

Corollary 4: For every real numbers $\mathfrak{d}_{0}$ and $\mathfrak{d}_{\infty}$ in $\left(-1, \frac{1}{n-1}\right)$, the origin of system (15) and (19) is a globally asymptotically stable equilibrium and the same holds for its homogeneous approximations.

\section{APPLICATION TO NONLINEAR SYSTEMS}

\section{A. Results on output feedback}

In this Section, we consider a system in the form :

$$
\dot{x}=\mathcal{S} x+B u+\delta(x, t), \quad y=x_{1},
$$

where $x=\left(x_{1}, \ldots, x_{n}\right)$ is in $\mathbb{R}^{n}$ and $\delta: \mathbb{R}^{n} \times \mathbb{R}_{+} \rightarrow \mathbb{R}^{n}$ is a continuous function.

The system (20) has the structure of a chain of integrators disturbed by nonlinear terms. So to design a stabilizing output feedback, we follow the domination approach introduced in [13] (see also [22]) where a linear controller was introduced to deal with a nonlinear system. This approach has also been followed with a nonlinear controller in [20] and in combination with weighted homogeneity in [27], [21], [23] and references therein.

In the domination context the nonlinear functions (the $\delta_{i}$ 's) are not used in the design but considered as perturbations and the controller is designed on a dominating model which in this case is the chain of integrators. To ensure robustness to these nonlinearities, we employ high-gain techniques. This leads us to work with the scaled coordinates :

$$
\mathcal{X}_{i}=L^{1-i} x_{i}, \quad i=1, \ldots, n,
$$

where $L$ is the high-gain parameter (a positive real number), or in compact form

$$
\mathfrak{X}=\mathfrak{L} x \quad, \quad \mathfrak{L}=\operatorname{diag}\left(1, L^{-1}, L^{-2}, \ldots, L^{1-n}\right),
$$

Employing the homogeneous in the bi-limit output feedback for a chain of integrator (Corollary 4), the small gain result (Corollary 2) in combination with high-gain technique we obtain an output feedback result. The output feedback we propose is given as :

$$
\dot{\hat{\mathfrak{X}}}=L\left(\mathcal{S} \hat{\mathfrak{X}}+B \phi(\hat{\mathfrak{X}})+K\left(\hat{\mathcal{X}}_{1}-y\right)\right), u=L^{n} \phi(\hat{\mathfrak{X}}),
$$

where $\phi$ and $K$ are given in Theorem 2 and 3 , and $L$ in $\mathbb{R}_{+}$ is the high-gain parameter.

Depending on wether $\mathfrak{d}_{0} \leq \mathfrak{d}_{\infty}$ or $\mathfrak{d}_{\infty} \leq \mathfrak{d}_{0}$ we get a result for systems in Feedback or Feedforward form.

Corollary 5 (Feedback form): If $\mathfrak{d}_{0} \leq \mathfrak{d}_{\infty}$ and

$$
\delta(x, t) \leq c_{0} \sum_{j=1}^{i}\left|x_{j}\right|^{\frac{1-\mathfrak{o}_{0}(n-i-1)}{1-\mathfrak{o}_{0}(n-j)}}+c_{\infty} \sum_{j=1}^{i}\left|x_{j}\right|^{\frac{1-\mathfrak{d}_{\infty}(n-i-1)}{1-\mathfrak{d}_{\infty}(n-j)}}
$$

where $c_{0}$ and $c_{\infty}$ are positive real numbers then there exist a real number $L^{*}>0$ such that for every $L$ in $\left[L^{*},+\infty\right)$, the origin of system (20) with the output feedback (22) is globally and asymptotically stable.

Corollary 6 (Feedforward form): If $\mathfrak{d}_{\infty} \leq \mathfrak{d}_{0}$ and

$\delta(x, t) \leq c_{0} \sum_{j=i+2}^{n}\left|x_{j}\right|^{\frac{1-\mathfrak{o}_{0}(n-i-1)}{1-\mathfrak{o}_{0}(n-j)}}+c_{\infty} \sum_{j=i+2}^{n}\left|x_{j}\right|^{\frac{1-\mathfrak{o}_{\infty}(n-i-1)}{1-\mathfrak{d}_{\infty}(n-j)}}$

where $c_{0}$ and $c_{\infty}$ are positive real numbers, there exists a real number $L^{*}>0$ such that for every $L$ in $\left(0, L^{*}\right]$, the following holds, the origin of system (20) with the output feedback (22) is globally and asymptotically stable.

The results presented in [1] are actually more general since the bound on the functions $\delta_{i}$ 's is expressed in terms of a small gain condition.

Employing a dynamic high-parameter, a similar approach has been employed to solve a tracking trajectory problem in [2].

\section{B. Result on finite-time observer design}

The domination approach has been also widely used to design observers for systems of the form (20). This approach has been popularized by high-gain observer [9]. These observers are given as:

$$
\dot{\hat{x}}=\mathcal{S} \hat{x}+B u+\delta(\hat{x}, t)+L \mathfrak{L}^{-1} K\left(\hat{x}_{1}-y\right)
$$

where $L$ is the high-gain parameter and $K$ the output injection which has to be designed to ensure that the state of the error system:

$$
\dot{\tilde{x}}=\mathcal{S} \tilde{x}+\delta(\tilde{x}, t)-\delta(\hat{x}-\tilde{x}, t)+L \mathfrak{L}^{-1} K\left(\tilde{x}_{1}\right)
$$


with $\tilde{x}=\left(\tilde{x}_{1}, \ldots, \tilde{x}_{n}\right)$ in $\mathbb{R}^{n}$ converges to the origin.

As previously, this system has the structure of a chain of integrators disturbed by nonlinear terms which assuming a global Lipschitz condition (as in [9]) is linearly bounded. In [9], the domination approach has been employed and a linear vector field $K$ in (23) was introduced to ensure global and asymptotic convergence of the error $\tilde{x}$ toward the origin.

Recently, this approach has been extended in [25] (see also [18]) to a homogeneous vector field $K$ with negative degree to allow semi-global and finite time estimation.

Employing the homogeneous in the bi-limit vector field $K$ obtained from Theorem 2 allows us to get a global observer with finite-time estimation and with an estimation time uniform in the initial condition.

Corollary 7 (Uniform Finite Time Observer): If $\exists c$ in $\mathbb{R}_{+}$such that

$$
\left|\delta_{i}(x+\tilde{x}, t)-\delta_{i}(x, t)\right| \leq c \sum_{j=1}^{i}\left|\tilde{x}_{j}\right|, \forall(x, \tilde{x}) \in \mathbb{R}^{2 n},
$$

then there exists a real number $L^{*}>0$ such that for every $L$ in $\left[L^{*},+\infty\right)$, the estimate given by the system (23) with $K$, the homogeneous in the bi-limit vector field obtained from Theorem 2 with $\mathfrak{d}_{0}<0<\mathfrak{d}_{\infty}$, converges to the state of system (20) in finite time uniformly in the initial condition.

The proof of this result is a direct consequence of [1] and can be found in [3].

Note however that this estimation algorithm is based on the integration of a non locally Lipschitz ordinary differential equation. In practice, this may cause some bad behavior of the resulting estimate.

Finally, the use of a dynamic high gain parameter allows to design a high-gain observer (not necessarily with finite time convergence) for some non-globally Lipschitz system as exposed in [4].

\section{CONCLUSION}

With the help of the notion of homogeneous approximation valid both at the origin and at infinity introduced in [1], we have reported results concerning asymptotic stability, robustness analysis and finite time convergence (uniformly in the initial conditions). We have then introduced a homogeneous in the bi-limit observer and controller for a chain of integrators. The combination of these two tools allows to obtain a new result on stabilization by output feedback for systems whose dominant homogeneous in the bi-limit part is a chain of integrators. A result concerning finite time convergent observers for globally Lipschitz system has also been given.

\section{REFERENCES}

[1] V. Andrieu, L. Praly, and A. Astolfi. Homogeneous approximation, recursive observer design and output feedback. SIAM Journal on Control and Optimization, 47(4):1814-1850, 2008.

[2] V. Andrieu, L. Praly, and A. Astolfi. Asymptotic tracking of a reference trajectory by output-feedback for a class of non linear systems. Systems \& Control Letters, 58(9):652-663, 2009.
[3] V. Andrieu, L. Praly, and A. Astolfi. High-gain observer with uniform in the initial condition finite time convergence. HAL document, 2009. URL:http:// hal.archives-ouvertes.fr/ /hal-00412585/ fr/.

[4] V. Andrieu, L. Praly, and A. Astolfi. High gain observers with updated gain and homogeneous correction terms. Automatica, 45(2):422-428, 2009.

[5] A. Bacciotti and L. Rosier. Liapunov Functions and Stability in Control Theory. Lecture Notes in Control and Information Sciences, 267, 2001.

[6] S.P. Bhat and D.S. Bernstein. Finite-time stability of continuous autonomous systems. SIAM Journal on Control and Optimization, 38(3):751-766, 2000.

[7] S.P. Bhat and D.S. Bernstein. Geometric homogeneity with applications to finite-time stability. Mathematics of Control, Signals, and Systems, 17(2):101-127, 2005.

[8] J.M. Coron and L. Praly. Adding an integrator for the stabilization problem. Systems \& Control Letters, 17(89):104, 1991.

[9] J.P. Gauthier and I. Kupka. Deterministic observation theory and applications. Cambridge University Press, 2001.

[10] H. Hermes. Homogeneous coordinates and continuous asymptotically stabilizing feedback controls. Differential Equations Stability and Control, 109:249-260, 1991.

[11] Y. Hong. Finite-time stabilization and stabilizability of a class of controllable systems. Systems \& control letters, 46(4):231-236, 2002.

[12] M. Kawski. Stabilization of nonlinear systems in the plane. Systems \& Control Letters, 12(2):169-175, 1989.

[13] H.K. Khalil and A. Saberi. Adaptive stabilization of a class of nonlinear systems using high-gain feedback. IEEE Transactions on Automatic Control, 32(11):1031-1035, 1987.

[14] S. Lefschetz. Differential equations: Geometric theory. New York, 1957.

[15] J.L. Massera. Contributions to stability theory. Annals of Mathematics, pages 182-206, 1956.

[16] P. Morin and C. Samson. Application of backstepping techniques to the time-varying exponential stabilisation of chained form systems. European journal of control, 3(1):15-36, 1997.

[17] R. Orsi, L. Praly, and I. Mareels. Sufficient conditions for the existence of an unbounded solution. Automatica, 37(10):1609-1617, 2001.

[18] W. Perruquetti, T. Floquet, and E. Moulay. Finite-Time Observers: Application to Secure Communication. IEEE Transactions on Automatic Control, 53(1):356-359, 2008.

[19] L. Praly, B. Andréa-Novel, and J.-M. Coron. Lyapunov design of stabilizing controllers for cascaded systems. IEEE Transactions on Automatic Control, 36(10):1177-1181, 1991.

[20] L. Praly and Z.P. Jiang. Stabilization by output feedback for systems with ISS inverse dynamics. Systems \& Control Letters, 21(1):19-33, 1993.

[21] C. Qian. A homogeneous domination approach for global output feedback stabilization of a class of nonlinear systems. In Proc. of American Control Conference, (ACC'05), pages 4708-4715, 2005.

[22] C. Qian and W. Lin. Output feedback control of a class of nonlinear systems: a nonseparation principle paradigm. IEEE Transactions on Automatic Control, 47(10):1710-1715, 2002.

[23] C. Qian and W. Lin. Recursive observer design, homogeneous approximation, and nonsmooth output feedback stabilization of nonlinear systems. IEEE Transactions on Automatic Control, 51(9):1457-1471, 2006.

[24] L. Rosier. Homogeneous Lyapunov function for homogeneous continuous vector field. Systems \& Control Letters, 19(6):467-473, 1992.

[25] Y. Shen and X. Xia. Semi-global finite-time observers for nonlinear systems. Automatica, 44(12):3152-3156, 2008.

[26] E.D. Sontag. Input to state stability: Basic concepts and results. Lecture Notes in Mathematics-Springer, 1932:163, 2008.

[27] B. Yang and W. Lin. Homogeneous observers, iterative design, and global stabilization of high-order nonlinear systems by smooth output feedback. IEEE Transactions on Automatic Control, 49(7):1069-1080, 2004 